\title{
Iron at the angle
}

Ch'ng Tun Wang, Ng Hong Kee

Department of Ophthalmology, Hospital Raja Permaisuri Bainun, Ipoh, Perak, Malaysia

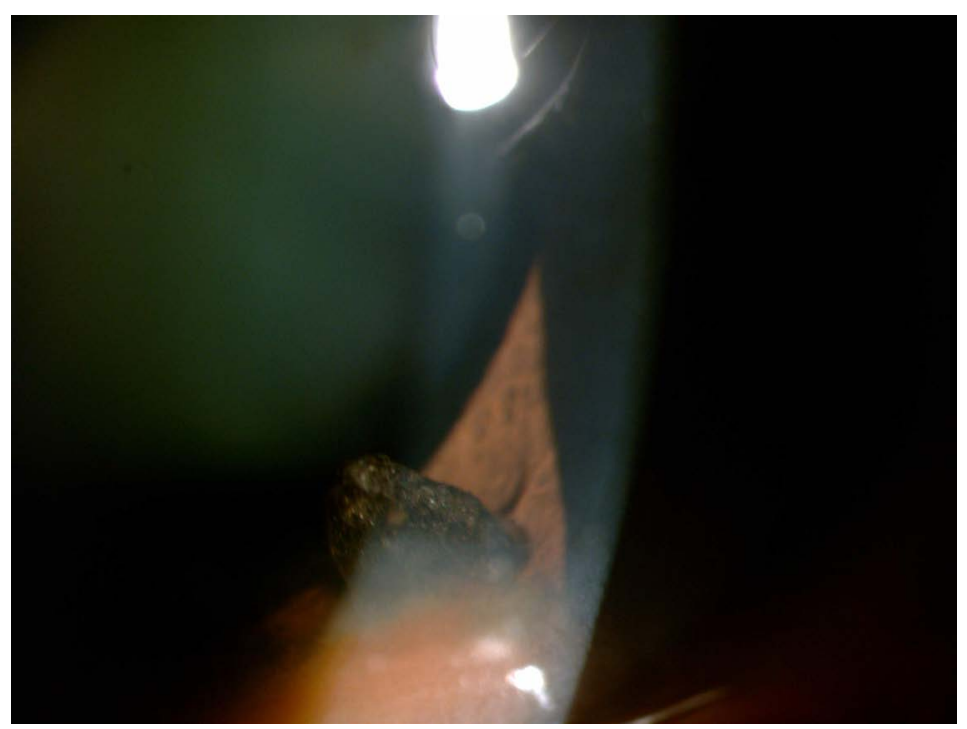

Fig. 1.

\section{Clinical context}

An incidental finding of a 55-years-old who man presented to eye clinic for conjunctivitis. Further assessment revealed history of ocular trauma 10 years ago while cutting grass. Vision in the right eye was $6 / 9$; the anterior chamber was quiet with normal fundus.

\section{Question 1}

Describe the findings in Figure 1.

Correspondence: Ch'ng Tun Wang, Department of Ophthalmology, Hospital Raja Permaisuri Bainun, Ipoh, Perak, Malaysia.

E-mail:chngtw@yahoo.com 


\section{Question 2}

What are the potential complications of a retained intraocular metallic foreign body?

\section{Answer 1}

A triangular shape of metallic foreign body seen at the inferior angle of the eye with localized metallic rust deposition on the adjacent endothelium.

\section{Answer 2}

The potential complications of ocular siderosis ${ }^{1}$ are the following:

- Cornea: Haziness and granular appearance of the corneal stroma with endothelium.

- Trabecular meshwork: Secondary open-angle glaucoma.

- Iris and pupil: Iris heterochromia, anisocoria, and accommodation failure.

- Lens: Focal rusty-brown nodules of subcapsular cataract and siderosis lentis.

- Retina: Degeneration of the inner retina and retinal pigment epithelium.

\section{References}

1. Hope-Ross M, Mahon GJ, Johnston PB. Ocular Siderosis. Eye. 1993;7;419-425. 